\title{
Relevancia de los resultados de pruebas de laboratorio para predecir la recuperación de hierro a nivel industrial
}

\author{
Luis I. Valderrama ${ }^{1 *}$, Oscar I. Herrera², y Ana M. Rivera ${ }^{1}$ \\ (1) Universidad de Atacama. Departamento de Ingeniería en Metalurgia. Copiapó, Chile. \\ (Correo-e: luis.valderrama@uda.cl; ana.rivera@uda.cl) \\ (2) Planta San José, Pucobre, Copiapó - Chile. (Correo-e: oscar.herrera@pucobre.cl;
}

Recibido Jun. 11, 2021; Aceptado Ago. 10, 2021; Versión final Sep. 14, 2021, Publicado Feb. 2022

\begin{abstract}
Resumen
Este trabajo tiene por objetivo evaluar la validez de los estimadores utilizados por la compañía CAP Minería para la determinación de las propiedades magnéticas de minerales de hierro. Se tomaron muestras de tres plantas ubicadas en la Región de Atacama (Chile): Cerro Negro Norte (CNN), Los Colorados y Planta Magnetita. Con equipos a escala de laboratorio, se realizaron concentraciones con el fin de optimizar los procesos de concentración magnética a nivel industrial. Los resultados de las leyes de Fe total, Femag, Fe DTT (Davis tube tester) y porcentaje de magnetismo muestran que la planta Magnetita es de bajo magnetismo y Los Colorados de alto magnetismo. Se aprecia un comportamiento uniforme en la recuperación en peso para las muestras de alto magnetismo provenientes de Planta CNN, Planta Magnetita y Los Colorados. Se concluye que la recuperación en peso calculada en base a magnetita sirve como estimador para la recuperación en peso global de la Planta CNN.
\end{abstract}

Palabras clave: magnetita; propiedades magnéticas; estimadores recuperación

\section{Importance of laboratory test results to estimate iron recovery at the industrial level}

\begin{abstract}
This study aims to assess the validity of estimators used by the mining company CAP Minería to determine iron ore magnetic properties. Samples were retrieved from three plants located in the Atacama Region (Chile): Cerro Negro Norte (CNN), Los Colorados, and Planta Magnetita. Sample concentration is performed by using laboratory-scale equipment to optimize magnetic concentration processes at the industrial level. The results of total Fe grade, Femag, Fe DTT (Davis tube tester), and magnetic percentage show that Planta Magnetita has low magnetic strength and that Los Colorados has high magnetic strength. There is uniform weight recovery for high magnetic samples obtained from CNN, Los Colorados, and Magnetita. It is concluded that a magnetite-based recovery weight calculation can be used as an estimator for global weight recovery at the CNN Plant.
\end{abstract}




\section{INTRODUCCIÓN}

La producción mundial de mineral de hierro el 2019 fue de 2.500 millones de toneladas, siendo la producción chilena de 14,5 millones de toneladas de mineral (principalmente mineral de magnetita) en sus diversas formas: pellets feed, pellets chips, finos y granza, con una ley de Fe promedio de $61,3 \%$. A nivel nacional, la Región de Atacama aportó la mayor cantidad de mineral de hierro $(87,9 \%)$, mientras que la Región de Coquimbo contribuyó con un 12,1\%. Los separadores magnéticos se basan en la diferencia de propiedades magnéticas entre los minerales magnéticos (magnetita y algunas hematitas) y las gangas no magnéticas, tales como cuarzo y feldespato (Valderrama, et al., 2020). Es por ello que los separadores magnéticos pueden clasificarse en equipos de baja y alta intensidad, y pueden agruparse en separadores de alimentación seca y de alimentación húmeda (Ge, et al., 2017).

Como la tendencia es aumentar la producción de hierro, se requerirán nuevos recursos de mineral de hierro, cuyos depósitos tendrán una menor ley de hierro y necesitarán una molienda más fina, para liberar los minerales de hierro. Por lo cual, en el proceso de separación magnética de los minerales de hierro de granulometría finas, las fuerzas magnéticas deben ser mayor a las fuerzas de arrastre hidrodinámicas y a las centrifugas. Las fuerzas que actúan sobre las partículas en un separador magnético son magnéticas, gravedad, resistencia hidrodinámica, fricción, inercia y centrífuga. La importancia relativa de cada fuerza varía con el diseño del separador. Sin embargo, las fuerzas de arrastre magnéticas, gravitacionales e hidrodinámicas son las principales fuerzas que gobiernan el comportamiento general de las partículas minerales en un separador magnético. Así, mientras las fuerzas magnéticas atraen partículas magnéticas, las fuerzas de gravedad y arrastre actúan contra las fuerzas magnéticas. Por otra parte, el tamaño de partícula de los minerales tiene un efecto pronunciado sobre la magnitud de estas fuerzas. De igual manera, se ha informado que las fuerzas de arrastre hidrodinámicas son proporcionales al diámetro de las partículas, mientras que las fuerzas magnéticas y de gravedad son proporcionales a la segunda y tercera potencia del diámetro de las partículas, respectivamente (Xiong, et al., 2015; Stener, 2016).

Las fuerzas magnéticas, gravitacionales e hidrodinámicas son las principales fuerzas que gobiernan el comportamiento general de las partículas minerales en un separador magnético. Es por ello que el tamaño de las partículas de los minerales tiene un efecto significativo sobre estas fuerzas. Por consiguiente, mientras las fuerzas de arrastre hidrodinámicas se vuelven más dominantes para las partículas finas, las fuerzas de gravedad son dominantes para las partículas gruesas y las fuerzas magnéticas para el rango de tamaño intermedio. En la práctica la separación magnética de minerales ferromagnéticos de tamaño de partícula inferior a $74 \mu \mathrm{m}$ no es muy efectiva (Stener, 2016). De hecho, Khokhulya, et al., (2021) indica que en la Planta Olcon, en Rusia, se recupera el hierro contenido en el relave mediante la separación magnética de las fracciones finas de magnetita de tamaño menor a $-71 \mu \mathrm{m}$, obteniendo concentrado magnético con leyes de hierro de $68 \%$ y recuperación de $86,7 \%$.

La práctica actual para evaluar la eficiencia de la recuperación de magnetita a nivel industrial es mediante la realización de pruebas a escala de laboratorio, por diversos métodos: tubo Davis, susceptómetro, medir el contenido de hierro por análisis químico, medir la susceptibilidad magnética y uso de la balanza de saturación magnética (Satmagan). El tubo Davis es un instrumento de laboratorio diseñado para separar pequeñas muestras de minerales magnéticos de minerales débilmente magnéticos. Se ha convertido en un equipo de laboratorio estándar utilizado para la evaluación de la separabilidad de minerales magnéticos mediante separadores magnéticos de baja intensidad. (Abdel Haffez. y Abu-Ali, 2012; Murariu, y Svoboda, 2003) sugirieron que se debe usar una inducción magnética de 0,4 T o más entre los polos magnéticos. Por otro lado, Stener, et al., (2016) afirman que la práctica actual es realizar las pruebas a una inducción magnética igual a la de la superficie del tambor del separador magnético. Esto es bastante cuestionable, en primer lugar, porque la eficiencia de la separación no está determinada por la intensidad del campo magnético, sino por el producto de la inducción magnética y el gradiente de campo; la segunda razón es que, para tener una recuperación eficiente del material magnético, en el tambor magnético se requiere una distancia suficiente entre el tambor y el fondo del estanque (Ahmed, 2010). Es bien sabido que el índice de fuerza disminuye rápidamente al aumentar la distancia desde la superficie del tambor, por lo cual resulta obvio que la información obtenida en las pruebas de tubo Davis no pueden ser directamente aplicable a los separadores magnéticos de tambor.

Otro equipo usado para cuantificar el contenido de magnetita es el Satmagan, en que las muestras de polvo son sometidas a un campo magnético suficientemente fuerte $(>2,2 \mathrm{~T})$ que permite la saturación magnética de minerales (Du Breuil, et al., 2015). Consiste en una pequeña celda de plástico (1,2 mL) que se llena con la muestra y se introduce en un campo magnético inducido por una bobina interna. La relación entre la fuerza gravitacional y la fuerza magnética es proporcional al contenido de mineral magnético en la muestra. El momento magnético de otros minerales es insignificante en comparación con los minerales magnéticos. Es un método no destructivo y rápido, cuyos resultados dependen de la calibración del equipo, realizada en diez muestras estándar certificadas. 
Como el mineral que está siendo procesado en la Planta CNN, disminuyo la ley de hierro, la relación para determinar el porcentaje de magnetita con pruebas de laboratorio solo se cumple para muestras con alto magnetismo, mientras que para las muestras de mediano y bajo magnetismo no existe relación, porque los factores de correlación obtenidos son bastante bajos, por lo tanto el objetivo de este trabajo es evaluar la validez de los estimadores que actualmente utiliza por CAP Minería, para determinar las propiedades magnéticas de minerales de hierro, utilizando equipos a escala de laboratorio, con la finalidad de obtener una mejor representación de éstas para los procesos industriales de la concentración magnética.

\section{MATERIALES Y MÉTODOS}

Las muestras de mineral utilizadas en este estudio fueron proporcionadas por la Compañía Minera del Pacífico (CMP), proveniente de la planta Cerro Negro Norte (CNN), Los Colorados y Planta Magnetita, todas ubicadas en la Región de Atacama, Chile. Estas muestras fueron recolectadas en la descarga del chancador terciario de granulometría $3 \mathrm{~mm}$ en cada planta, reducidas de tamaño hasta granulometría menor a $150 \mu \mathrm{m}$; de esta manera se estandarizaron todas las muestras en un solo perfil granulométrico, con la finalidad de que las pruebas subsiguientes puedan seguir un procedimiento común. Cada muestra fue homogenizada y mediante cuarteos sucesivos para obtener muestra para los diferentes ensayos a realizar. Las muestras correspondientes $\mathrm{CNN}$ fueron recolectadas para cumplir con las distribuciones de ley de hierro magnético (\% Femag.) y magnetismo (M), descritas en la Figura 1. De igual manera, en la Tabla 1 se presenta la matriz de distribución del total de muestras según los aspectos mencionados.

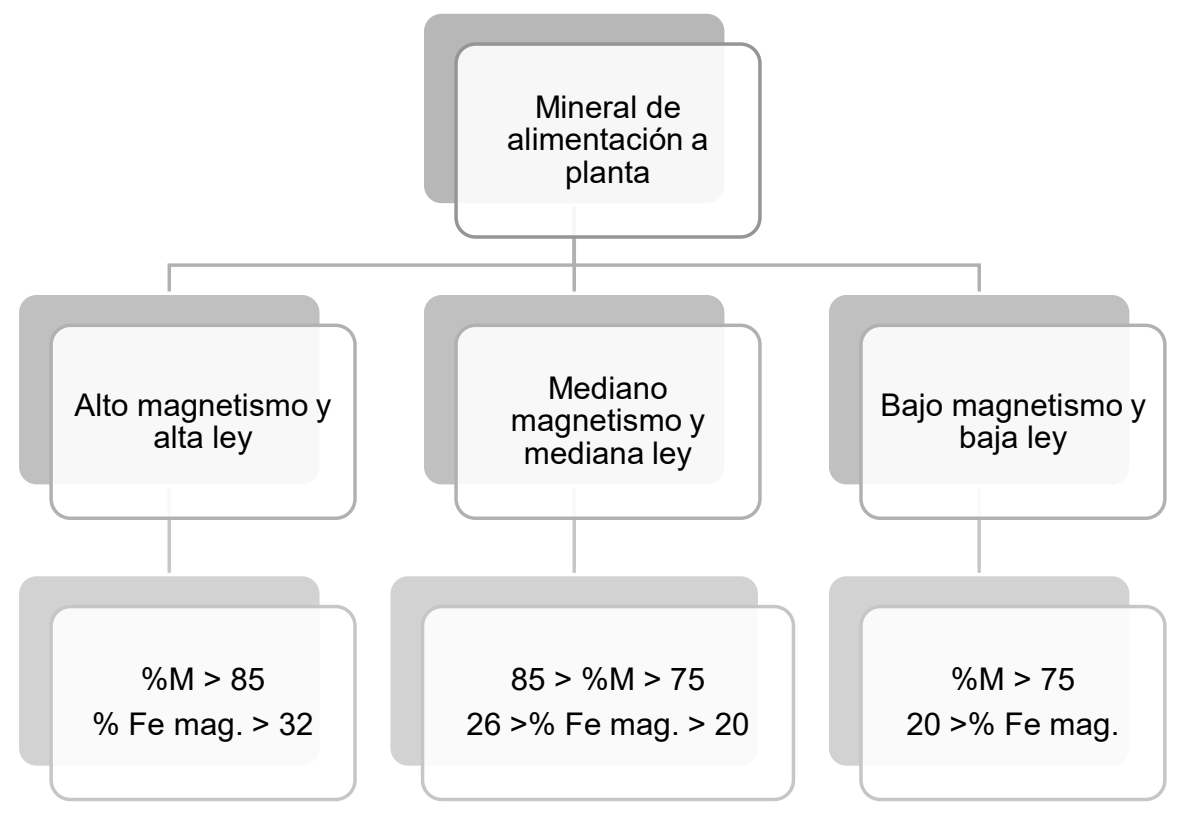

Fig. 1: Distribuciones de ley de hierro magnético y magnetismo

Tabla 1: Matriz de distribución de muestras Planta Cerro Negro Norte

\begin{tabular}{|c|c|c|c|}
\hline \% Fe mag. & Alto & Medio & Bajo \\
\hline Alta & 8 & 5 & 1 \\
\hline Media & 5 & 5 & 1 \\
\hline Baja & 3 & 1 & 2 \\
\hline
\end{tabular}

La homogenización de muestras se realizó mediante la técnica de cono y cuarteo, hasta la obtención de una submuestra de aproximadamente 400 gramos, la que se redujo de tamaño utilizando un pulverizador de anillos, hasta lograr la granulometría deseada determinada por los diferentes métodos de análisis granulométricos. Se realizaron análisis químicos a las muestras de cabeza tanto como para los concentrados obtenidos. Para la determinación de hierro total y óxido de hierro se utilizaron las técnicas de volumetría del dicromato de potasio, descrita por Hu, et al., (2014). 
Para la determinación de magnetita presente en las muestras, se utilizó la relación estequiométrica existente entre el $\mathrm{FeO}$ en relación directa con el $\mathrm{Fe}_{3} \mathrm{O}_{4}$ (magnetita), la cual puede ser calculada de la forma indicada en la ecuación 1. El análisis tubo Davis consistió en un tubo de vidrio de $30 \mathrm{~mm}$ de diámetro inclinado $45^{\circ}$, dentro del cual se adicionaron 20 gramos de muestra acondicionada como pulpa. Un sistema de bobinas colocadas en ambos lados del tubo proporcionó un campo magnético regulable de alta intensidad, llegando a alcanzar hasta 0,4 Tesla. Su campo magnético produjo la concentración de partículas magnéticas basada en la susceptibilidad magnética de las muestras, la cual midió la cantidad de mineral ferromagnético presente. Esta medición corresponde a la recuperación en peso del mineral obtenida en el separador magnético tubo Davis. Las diferentes muestras se analizaron individualmente como alimentación y concentrado DTT mediante análisis químico para la determinación de diferentes compuestos ( $\mathrm{Fe}, \mathrm{FeO}, \mathrm{SiO}_{2}, \mathrm{CaO}, \mathrm{MgO}, \mathrm{Al}_{2} \mathrm{O}_{3}, \mathrm{~N}_{2} \mathrm{O}$, $\mathrm{K}_{2} \mathrm{O}$ ). Los análisis en tubo Davis permitieron determinar la recuperación en peso, ley de hierro magnético y magnetismo. En la Tabla 2 se presentan los parámetros utilizados para estos análisis.

Tabla 2: Parámetros operacionales para ensayo DTT

\begin{tabular}{|l|c|}
\hline Parámetros & Ensayo \\
\hline Peso de muestra, $(\mathrm{g})$ & 20 \\
\hline Granulometría de muestra $(\mu \mathrm{m})$ & $85 \%-44 \mu \mathrm{m}$ \\
\hline Intensidad campo magnético, $(\mathrm{T})$ & $0,1-0,4$ \\
\hline Agua de lavado, $(\mathrm{mL} / \mathrm{min})$ & 600 \\
\hline Golpes por minuto & 110 \\
\hline Tiempo de ensayo, $(\mathrm{min})$ & 10 \\
\hline Angulo de inclinación, $\left({ }^{\circ}\right)$ & 45 \\
\hline
\end{tabular}

La cantidad de magnetita presente en las muestras se determinó mediante balanza de saturación magnética, utilizando una granulometría de mineral de $100 \%-149 \mu \mathrm{m}$, utilizando 1 a 2 gramos o $1,2 \mathrm{~mL}$ de muestra. Así, cuando se introduce al equipo la celda con muestra de mineral se realiza la primera medición, aplicando primero un campo gravitacional y luego un campo magnético creado por un imán permanente. Entonces, al llevar la balanza al equilibrio, la lectura entrega de forma directa el porcentaje en peso de magnetita de la muestra (ecn. 1).

$$
\% \mathrm{Fe}_{3} \mathrm{O}_{4}=\frac{\text { Peso molecular de } \mathrm{Fe} 3 \mathrm{O} 4}{\text { Peso molecular de } \mathrm{FeO}} \times \% \mathrm{FeO}
$$

\section{RESULTADOS Y DISCUSIÓN}

En la Tabla 3 se presenta la caracterización de las muestras provenientes de planta Magnetita y Los Colorados. Se observa que las leyes de Fe total, Femag, Fe DTT y \% de magnetismo son muy diferentes, indicando que planta Magnetita es de bajo magnetismo y Los Colorados de alto magnetismo.

Tabla 3: Caracterización de muestras provenientes de Planta Magnetita y Los Colorados.

\begin{tabular}{|l|c|c|}
\hline & Planta Magnetita & Los Colorados \\
\hline Fe (\%) & 10,16 & 41,00 \\
\hline Fe mag. (\%) & 6,18 & 38,30 \\
\hline Fe DTT (\%) & 67,90 & 70,47 \\
\hline Magnetismo (\%) & 60,82 & 93,41 \\
\hline
\end{tabular}

En la Figura 2 se presenta la caracterización de ley de hierro y FeO para el total de las muestras provenientes de planta CNN. La figura presenta las 31 muestras provenientes de la planta CNN que se le realizaron ensayos análisis químico de óxido ferroso, este análisis considera el ion ferroso presente en la muestra, pero en el ensayo del dicromato se detecta el ion ferroso de minerales, tales como, magnetita, pirita, actinolita, clorita que están presente en la muestra, por lo que al realizar el cálculo estequiométrico de porcentaje de magnetita, este porcentaje se ve sobreestimado debido a lo anteriormente mencionado, pero aun así, se considera que la cantidad de magnetita presente en la muestra de Planta Magnetita, 
En la Figura 3 se puede apreciar un comportamiento uniforme en la recuperación en peso para las muestras de alto magnetismo provenientes de Planta CNN, Planta Magnetita y Los Colorados. Para las muestras de medio y bajo magnetismo de Planta CNN, se observan diferencias en la recuperación en peso en el intervalo de 0,1 y 0,2 (Tesla), de $12,30 \%$ y $13,80 \%$, respectivamente. Esto se vuelve relevante porque los concentradores magnéticos utilizados industrialmente en Planta CNN, poseen intensidades de campo magnético entre 0,075 a 0,1 (T), por lo que las diferencias observadas en los ensayos DTT, se pueden ver replicados al momento de alimentar un circuito industrial con minerales de estas características.

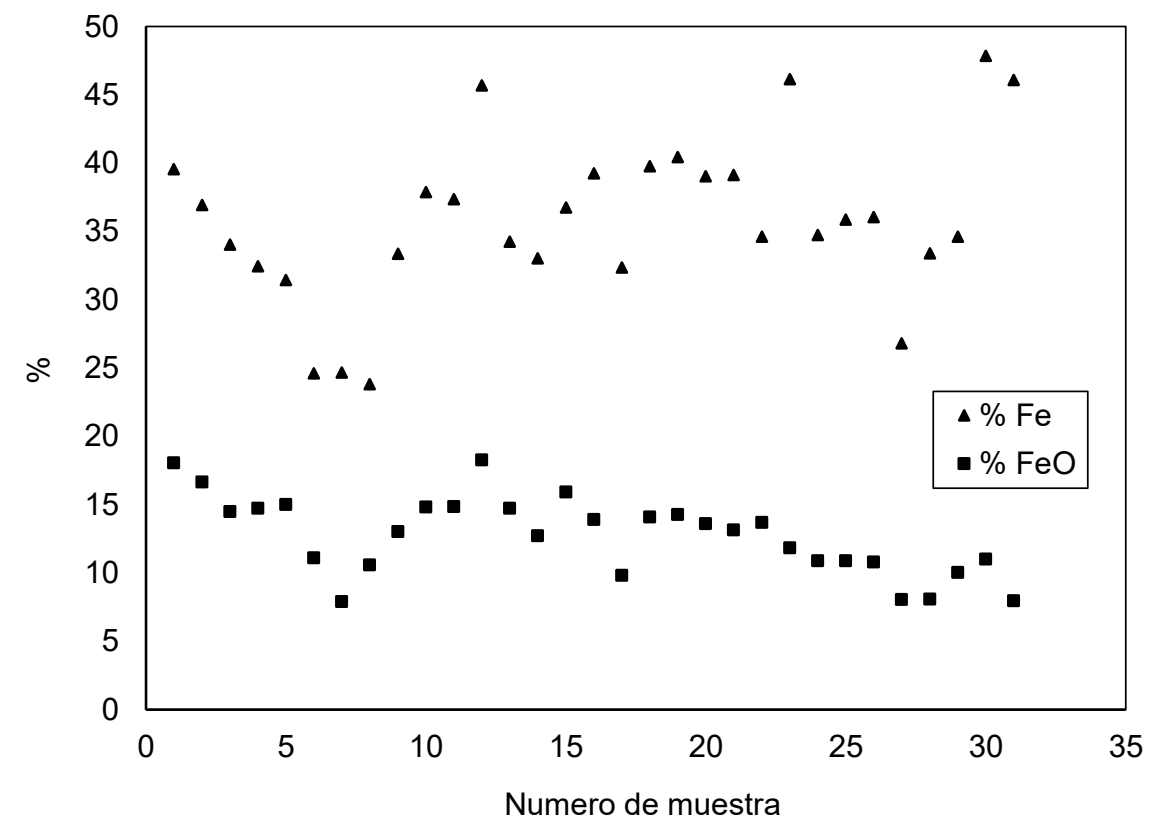

Fig. 2: Caracterización muestras provenientes de planta CNN

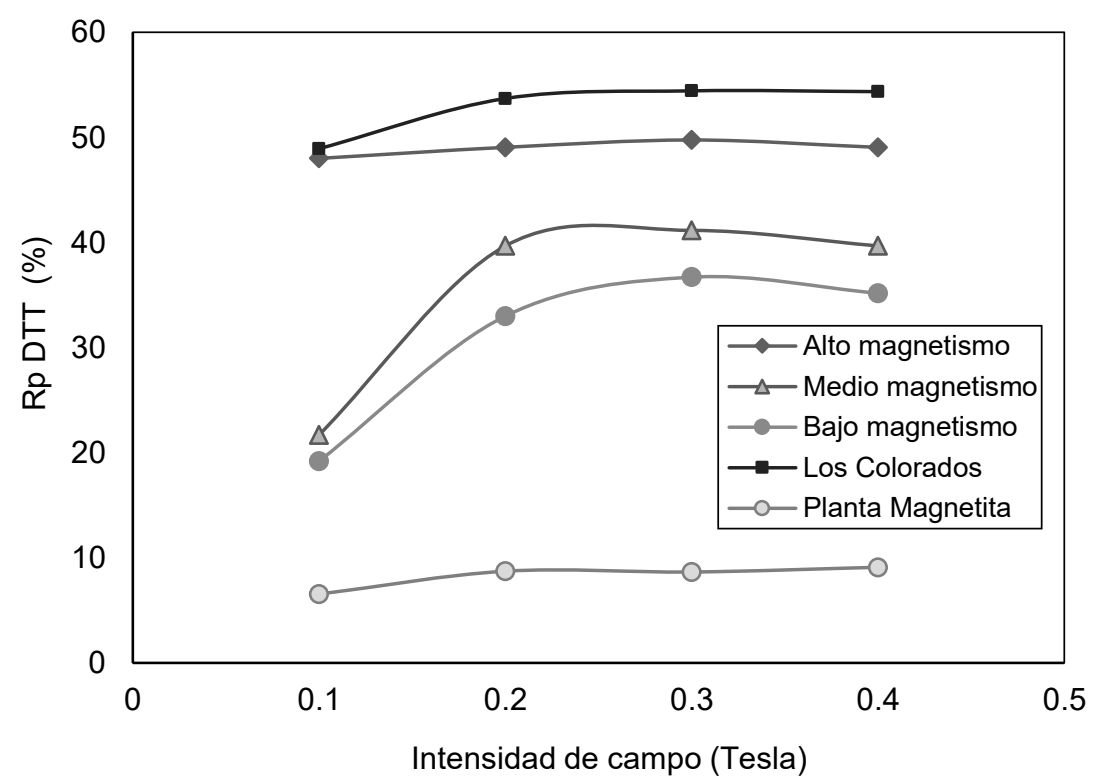

Fig. 3. Efecto de la intensidad del campo magnético en la recuperación en peso

Por otro lado, en la Figura 4 tal como lo observado en la figura anterior las leyes de hierro magnético se mantienen constantes para las muestras de alto magnetismo de Planta CNN junto con las muestras de Planta Magnetita y Los Colorados, mientras que para las muestras de mediano y bajo magnetismo se aprecian grandes diferencias en su comportamiento. Estos resultados permiten deducir que la ley de corte de hierro magnético de alimentación a planta es de $20 \%$ de Femag. Es por ello que al momento de considerar una ley de hierro magnético con respecto al ensayo en tubo Davis a 0,1 [T] de intensidad de campo magnético, estas muestras serían descartadas y consideradas como material estéril. 
En la Figura 5 se presenta una gráfica comparativa entre el análisis Satmagan y la cantidad de magnetita presente en las muestras, calculada de forma estequiométrica a través del análisis químico de óxido de hierro ( $\mathrm{FeO}$ ). Allí se observa que ambos métodos son adecuados para la determinación de magnetita presente en una muestra, tal como lo indica la teoría, puesto que presentan un coeficiente de correlación de 0,9345.

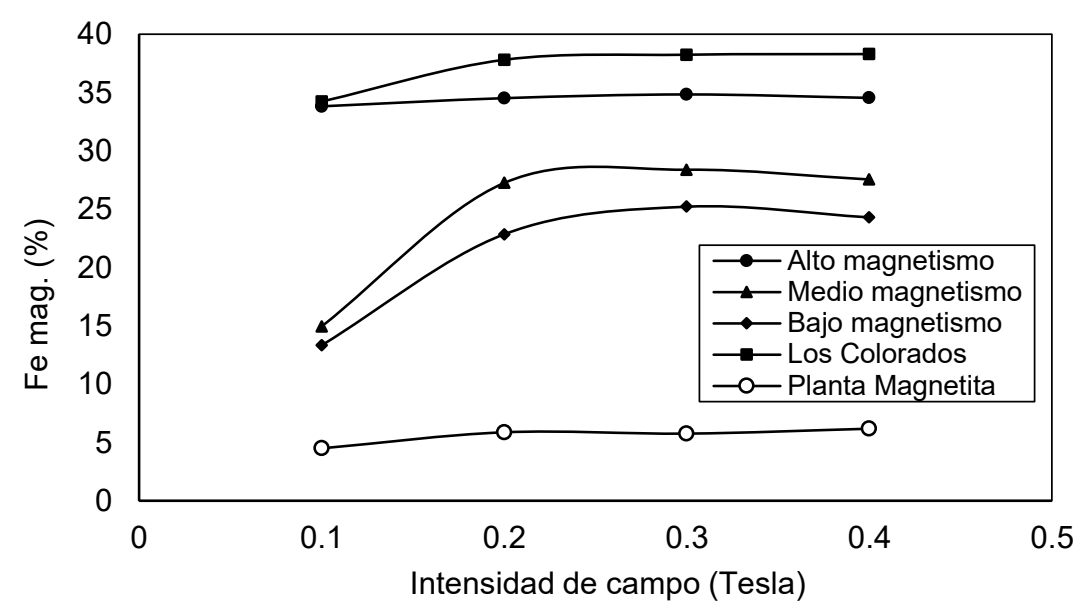

Fig. 4. Intensidad de campo magnético versus ley de hierro magnético.

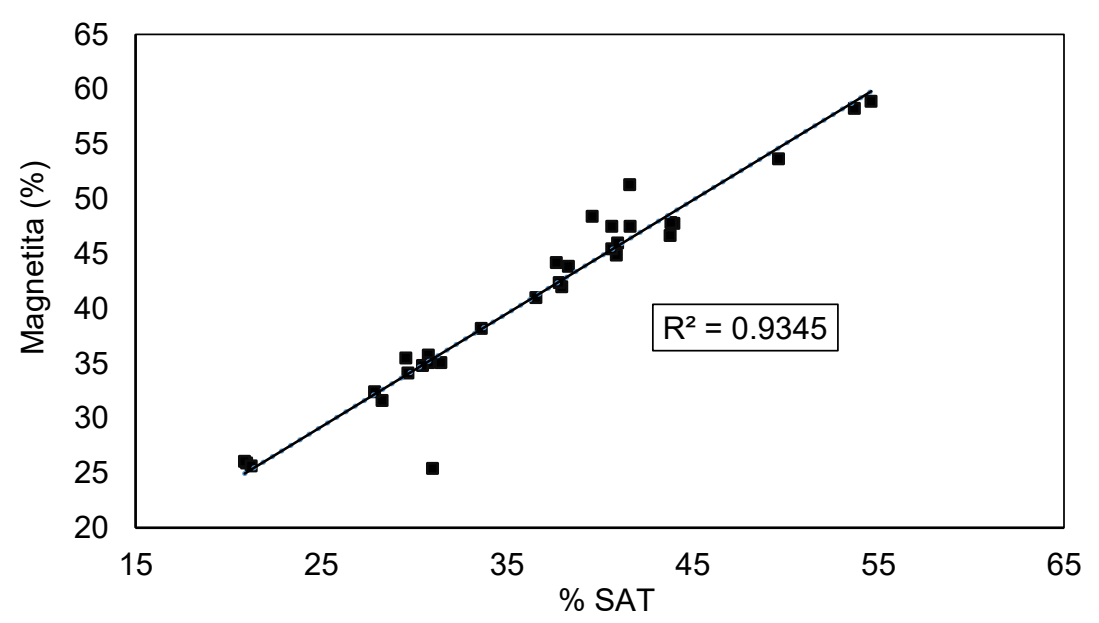

Fig. 5. \% SATMAGAN en función de la cantidad de magnetita presente en las muestras

En la Figura 6 se presenta el total de las muestras según su distribución de magnetismo que estas poseen. Así, para las muestras que presentan un alto magnetismo, efectivamente se mantiene la relación entre el ensayo Davis Tube Tester (DTT) y Satmagan, obteniendo un coeficiente de correlación de 0,9566, mientras que para las muestras de mediano y bajo magnetismo no existe tal relación, ya que los coeficientes de correlación son de 0,5625 y 0,1025, respectivamente. Esto está de acuerdo con lo informado por (Du Breuil, et al., 2015), que la eficiencia de separación magnética cambia dramáticamente en un nivel a otro, y ellos lo correlacionan con la mineralogía modal QEMSCAN, los análisis químicos de hierro y la magnetita obtenido por Satmagan, en este caso existen otros óxidos de hierro aparte de la magnetita. Al analizar la Figura 7 se puede observar que independientemente de la intensidad de campo magnético que se aplique en el ensayo DTT, se obtienen leyes de hierro similares para la mayoría de las muestras analizadas. Esto reafirma el propósito inicial del ensayo tubo Davis, el cual consistía en estimar leyes de hierro en el concentrado para los procesos de concentración magnética.

Por otra parte, en la Figura 8 se presenta el resultado de los análisis químicos de las impurezas presentes en el concentrado DTT para el total de las muestras estudiadas. Es posible observar que se presentan mayores cantidades de $\mathrm{SiO}_{2}$ en las muestras de alto magnetismo, mientras que en el resto de las muestras su contenido de sílice varía en función del magnetismo que presenten. Con relación al resto de impurezas, se verifica que se mantienen constantes independientemente en todo el rango de magnetismos, lo que hace inferir que las impurezas, exceptuando $\mathrm{SiO}_{2}$, pueden estar presentes en minerales de hierro que son concentrados en el ensayo tubo Davis. Estudio realizado por; Dworzanowski, (2012) en tubo Davis, muestran que, si la magnetita se encuentre más fina, el grado de liberación debe ser mayor; para obtener concentrados de hierro de mayor 
calidad, por lo cual deben ser molida a una granulometría menor a -10 $\mu \mathrm{m}$, pero a este tamaño granulométrico se produce una mayor selectividad para partículas liberadas de magnetita pero una reducción de la recuperación de masa de concentrado, y por lo tanto una menor probabilidad de arrastre de silicato. Lund, et al., (2013) indica que las muestras minerales deben ser caracterizado mediante análisis de microsonda electrónica (EPMA), fluorescencia de rayos X (XRF), Satmagan y QEMSCAN; pero estas técnicas no son rápida y económica para cuantificar minerales a partir de ensayos y ser usada como ensayos químicos de rutina.

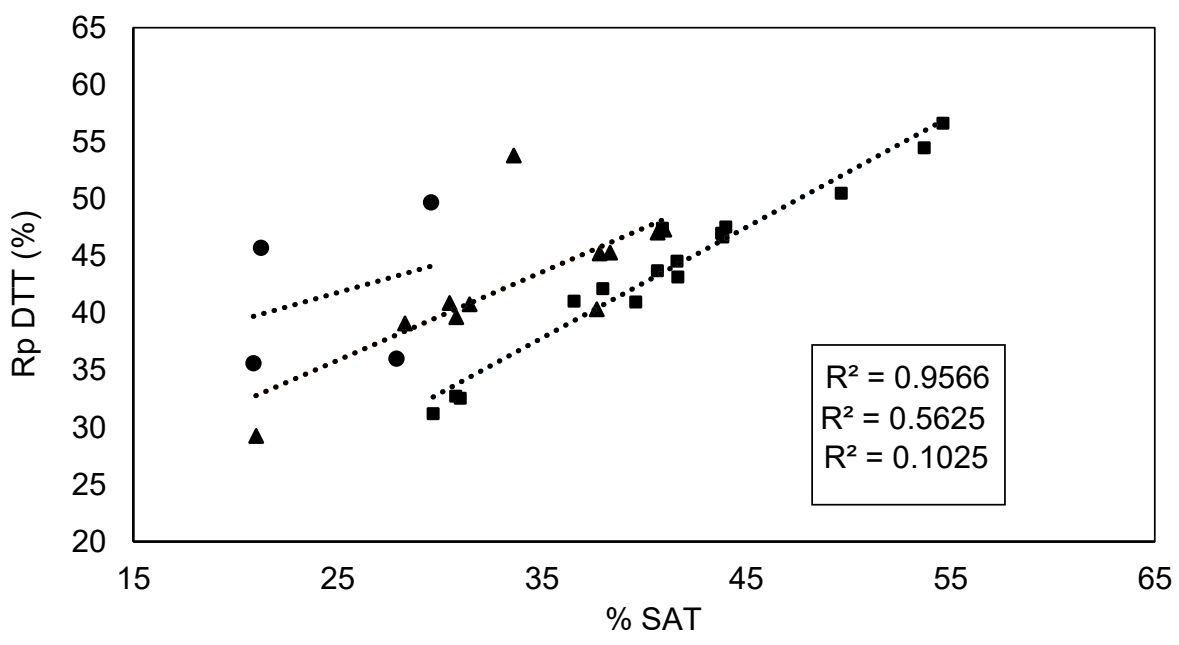

Fig. 6. \% SATMAGAN en función de la recuperación en peso

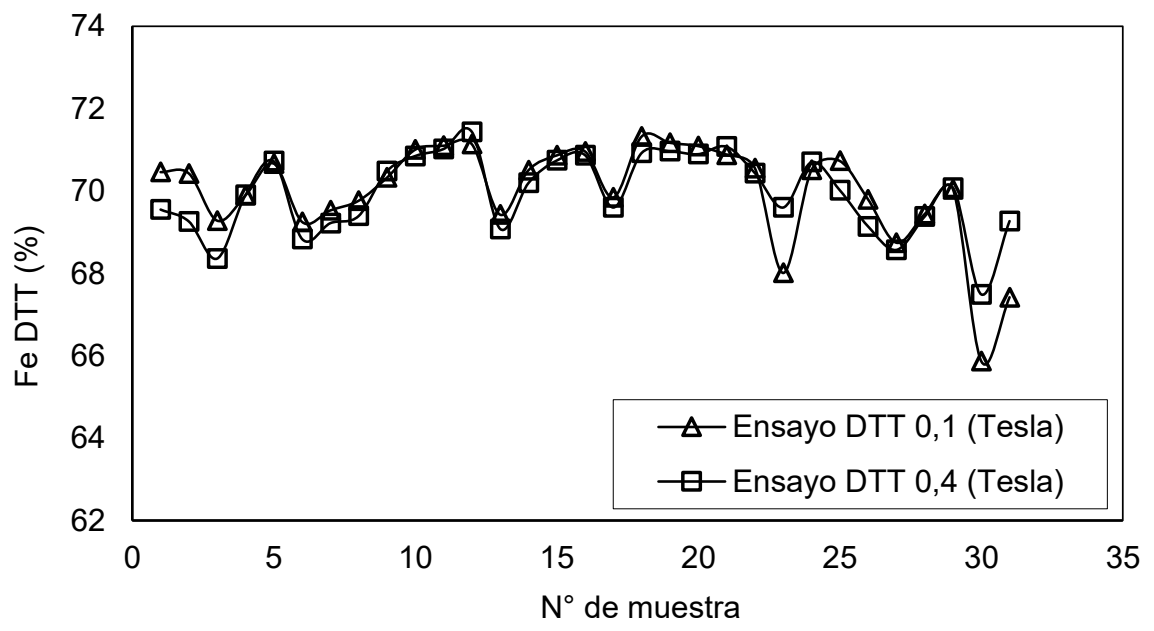

Fig. 7. Ley de hierro en el concentrado DTT obtenido con diferentes intensidades de campo magnético

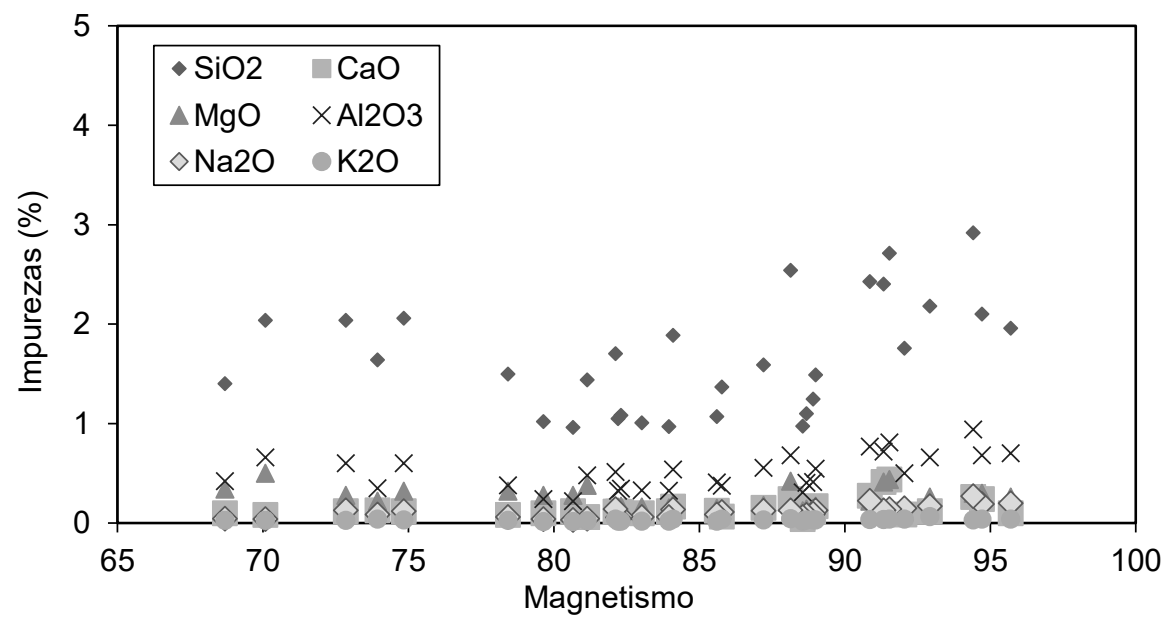

Fig. 8. Impurezas presentes en el concentrado DTT realizado a 0,4 Tesla de intensidad. 
Mediante análisis químicos por: $\mathrm{FeO}, \mathrm{SiO}_{2}, \mathrm{CaO}, \mathrm{MgO}, \mathrm{Al}_{2} \mathrm{O}_{3}, \mathrm{Na}_{2} \mathrm{O}$ y $\mathrm{K}_{2} \mathrm{O}$, se realizó una aproximación a la composición química de los concentrados DTT, calculando de forma estequiométrica la cantidad de magnetita en conjunto con las impurezas. En la figura 9 se presenta la recomposición de los concentrados obtenidos con respecto al magnetismo que originalmente poseían. De esta forma se puede observar que, para las muestras de alto magnetismo, sobre el $90 \%$ del concentrado corresponde a magnetita e impurezas, mientras que a medida que el magnetismo disminuye, la participación de estas especies se ve disminuida, esto es indicado por Issa et al., (2013).

En la figura 10 se puede apreciar la caracterización de las 27 muestras obtenidas desde planta CNN, se puede observar que corresponden a muestras de alta ley de hierro magnético (\% Fe mag) y altos magnetismos, donde se deja en claro que manteniendo altas calidades en el mineral alimentado se evita de cierta forma las diferencias en los balances de concentrado obtenido, esto fue indicado Ersayin (2004) mediante un modelo empírico basado en la relación ley de alimentación, tamaño de partícula y recuperación de magnetita.

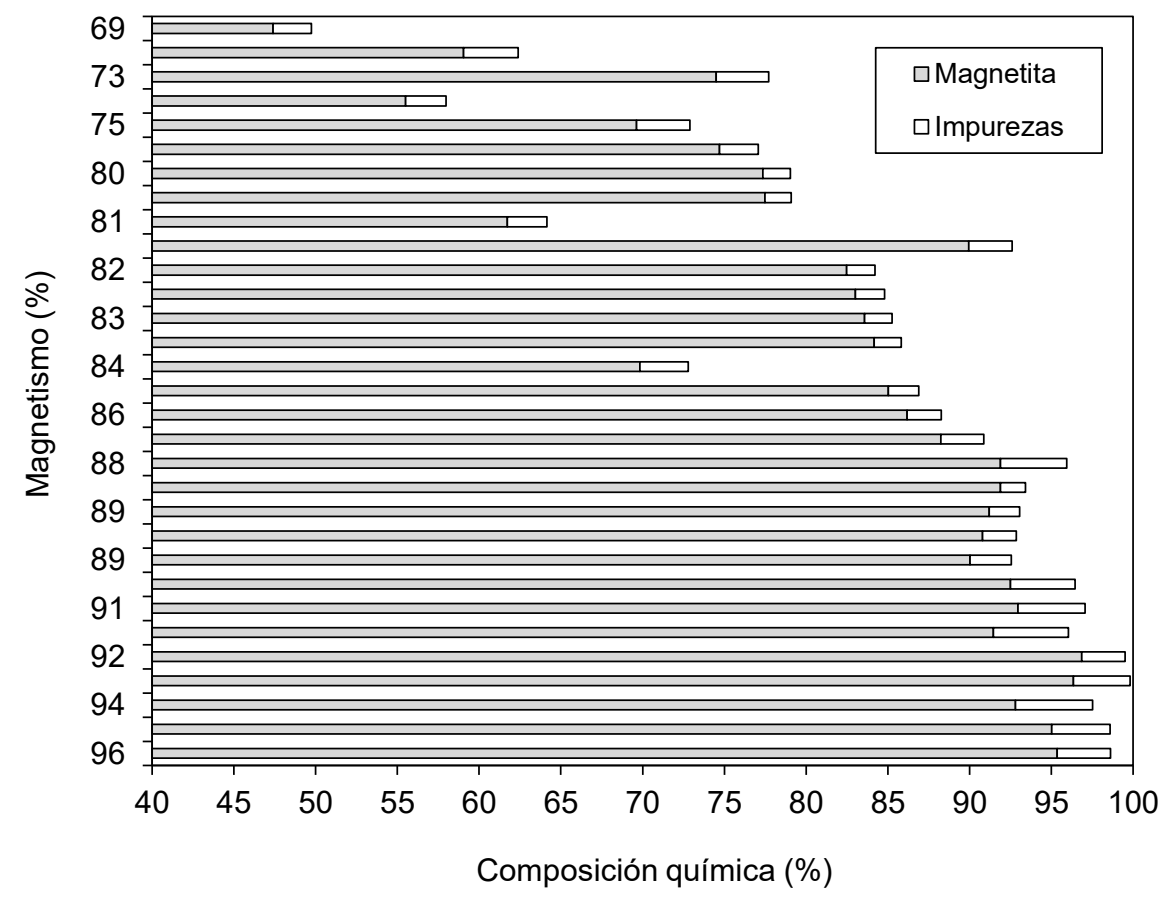

Fig. 9. Recomposición química de los concentrados DTT obtenidos utilizando los diferentes análisis químicos realizados

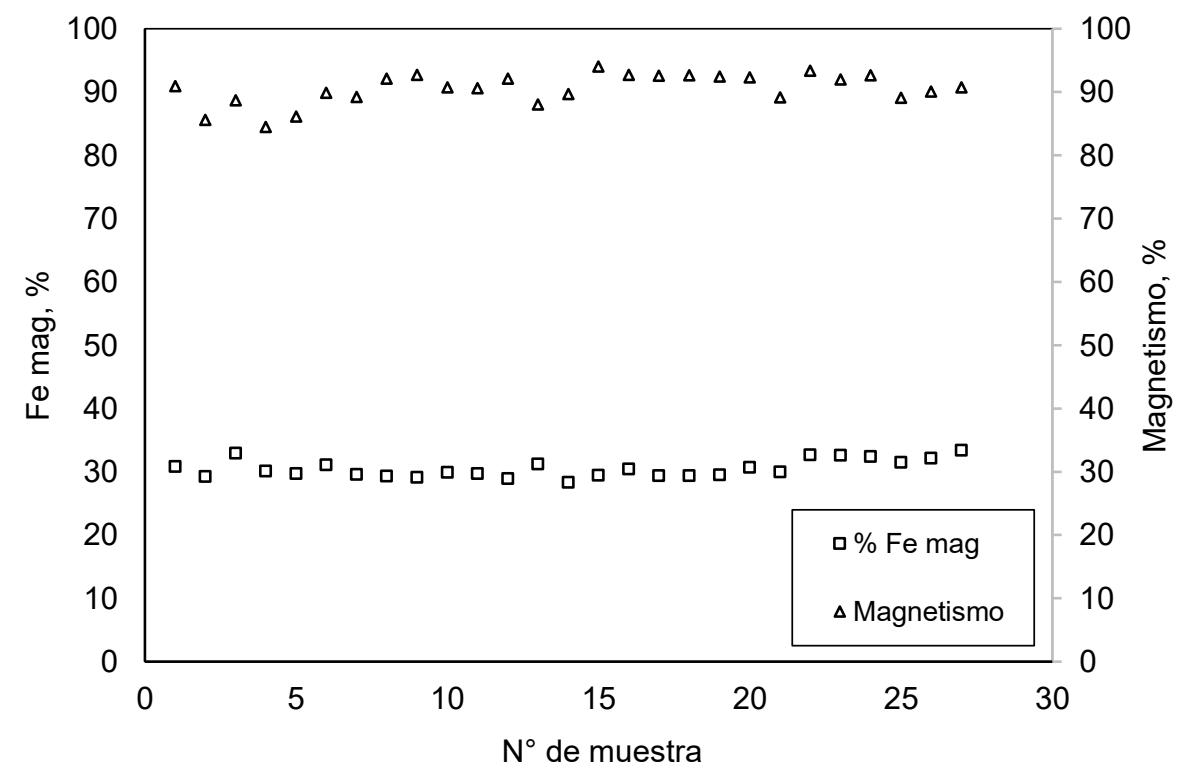

Fig. 10: Ley de hierro magnético y altos magnetismos para cada muestra de planta CNN 
Considerando los análisis de las figuras 6 y 7 , cuyas leyes de hierro en el concentrado DTT superan el $66 \%$, con contenido de impurezas que se mantienen constantes, se infiere que el resto del concentrado DTT que no corresponde a magnetita e impurezas, podrían contener otras especies de hierro de leyes similares, las cuales estarían siendo concentradas al momento de realizar el ensayo DTT (Stener et al., 2016). Basándose en las consideraciones antes indicadas, se pudo verificar que el comportamiento de una muestra no necesariamente estará ligado a las propiedades magnéticas que posea, es decir, cantidad de hierro magnético (\% Femag.) o magnetismo (Dwari, et al., 2013). Por lo tanto, se plantea una recuperación en peso en base a la cantidad de magnetita presente en el concentrado DTT, utilizando análisis químico de óxido de hierro. Dicha recuperación en peso, en base a magnetita, se presenta en la ecuación 2 , que considera un balance entre la recuperación en peso obtenida en el ensayo DTT con respecto a la cantidad de magnetita presente en el concentrado. Así, se considera el comportamiento individual de cada muestra, lo que podría evitar errores cuando se calcula una recuperación en peso en base a propiedades magnéticas.

$$
R_{\text {Pmagnetita }}=\left(\frac{F e O_{C D T T} \times C_{1} \times R_{P D T T}}{100 \%}\right)
$$

Para las constantes descritas se considera $C_{1}=3,2226$ como factor estequiométrico, mientras que $\mathrm{C}_{2}=1,8 \%$ como factor de impurezas, el cual representa a las impurezas de los concentrados Pellet Feed producidos por Planta CNN. Este parámetro representa un factor operacional real, y dado que está sujeto a condiciones industriales, tiende a ser mayor que las impurezas determinadas en el ensayo tubo Davis. La Figura 11, presenta la recuperación global de la planta, y se compara con la recuperación estimada con la recuperación obtenida a través del ensayo DTT. En efecto, el ensayo DTT sobreestima, en la mayoría de los casos, a la recuperación en peso que se obtiene finalmente en el circuito de planta CNN, esto es indicado por Stener et al., (2016). De igual manera, la estimación actualmente utilizada para determinar la recuperación a nivel planta, corresponde a un ajuste lineal a la curva entregada por los diferentes puntos obtenidos a través del ensayo DTT, en donde se observa una reducción en el valor de recuperación en peso, pero se mantiene la misma tendencia que posee el ensayo DTT.

En la Figura 12, se presenta una comparación entre la recuperación global de planta con respecto a la estimación realizada por la ecuación, y la recuperación en peso en base a magnetita, donde en la mayoría de los casos, la recuperación en peso propuesta se correlaciona de mejor forma con la recuperación en peso global obtenida por planta, resultados similares han sido informados por Baawuah et al., (2020).

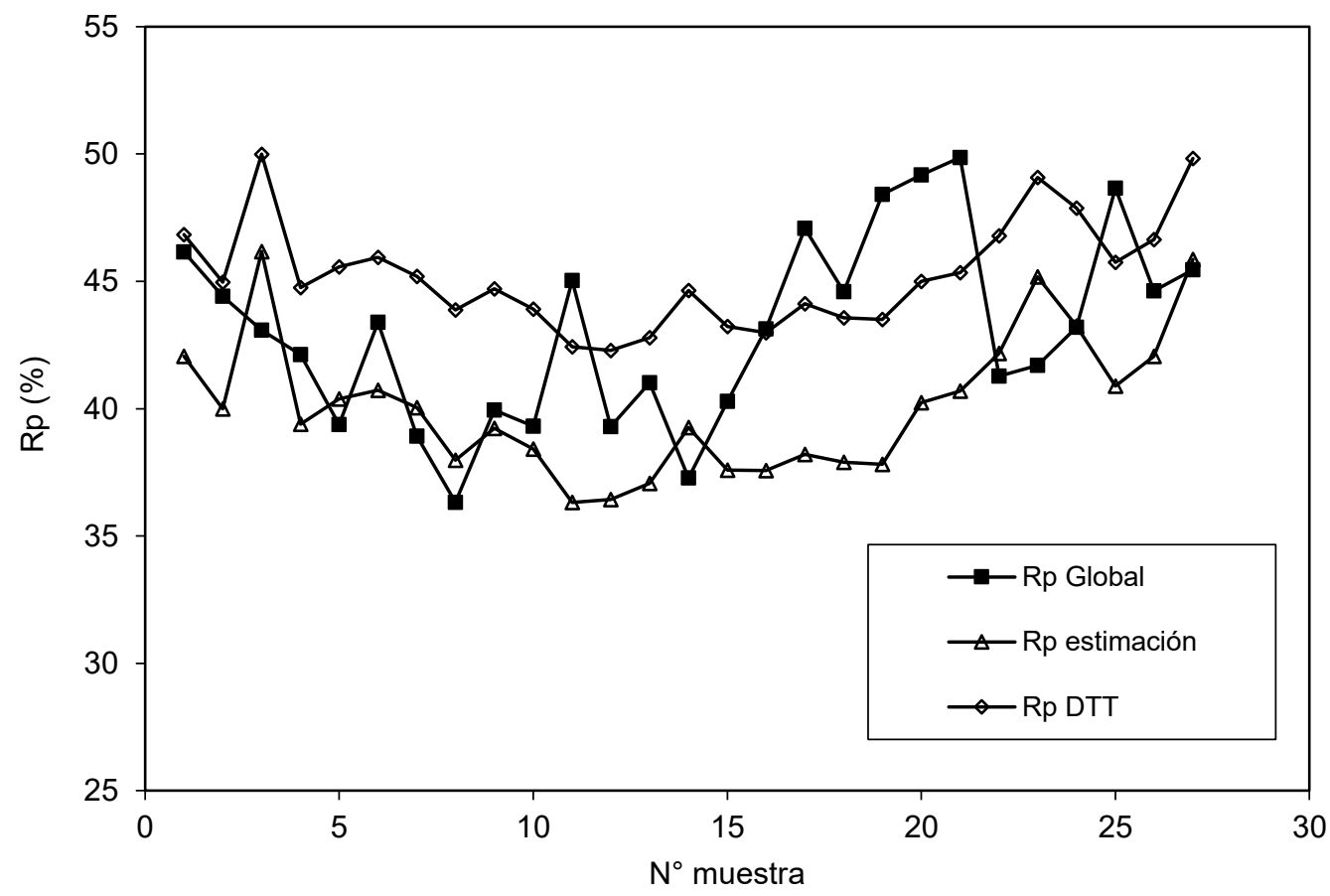

Fig. 11: Comparación entre recuperación en peso global de planta con respecto al ensayo DTT y estimación 


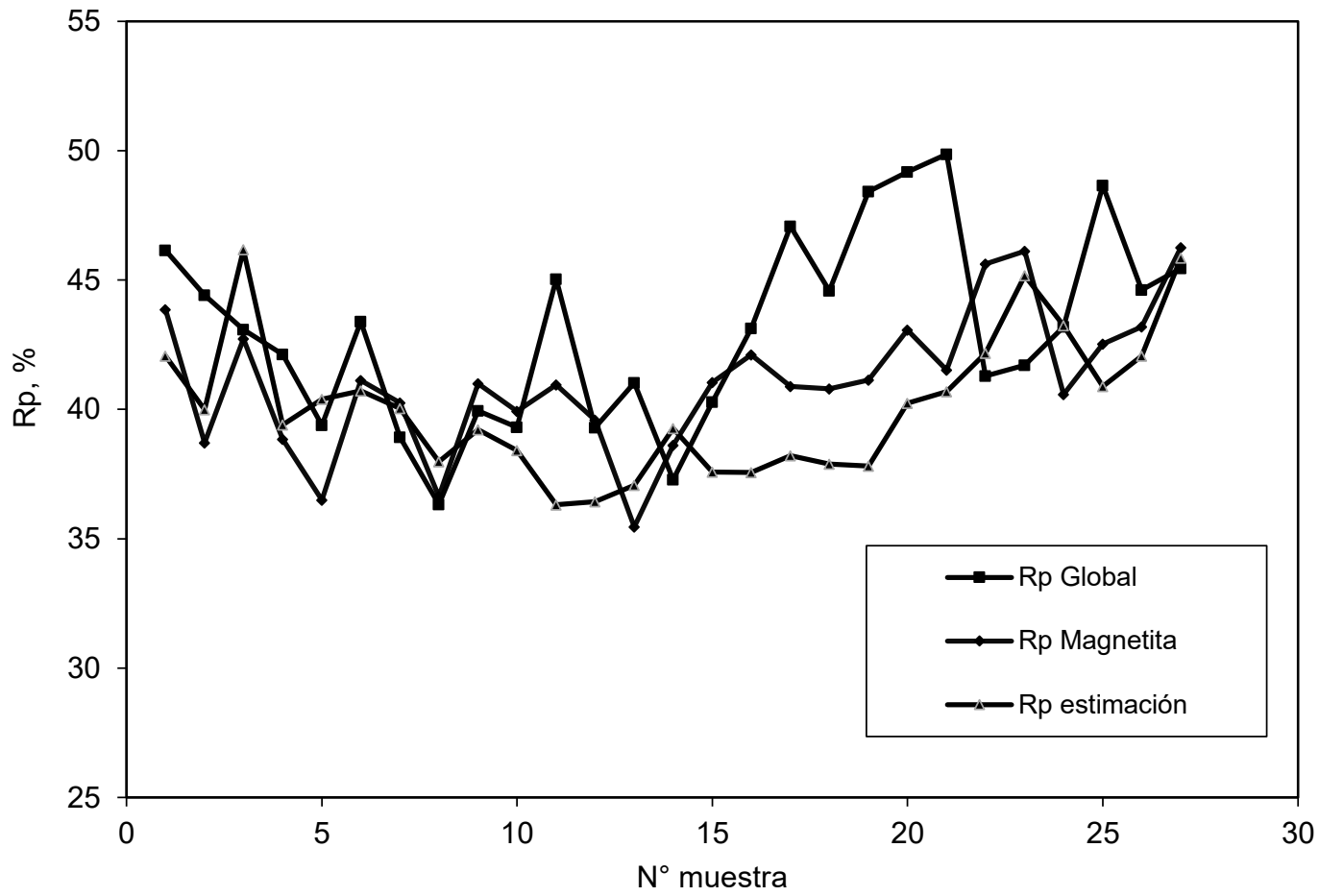

Fig. 12: Comparación entre recuperación global de planta con respecto a la estimación actualmente utilizada, y recuperación en peso en base a magnetita

Para efecto de comparación, en la Tabla 4 se presentan la desviación media absoluta junto con el error medio cuadrático y error medio absoluto para los diferentes métodos evaluados en la estimación de recuperación en peso, donde para el caso de la desviación absoluta media y el error medio absoluto se observa que la recuperación propuesta en base a magnetita presenta menores valores. Con esto se puede inferir que efectivamente es posible obtener un parámetro de recuperación en peso, que relaciona la información que los diferentes ensayos son capaces de entregar

Tabla 4: Comparación entre los diferentes métodos utilizados para el cálculo de recuperación.

\begin{tabular}{|l|c|c|c|c|c|}
\hline & $\begin{array}{c}\text { Rp DTT 0,4 } \\
(T)\end{array}$ & $\begin{array}{c}\text { Rp DTT } \\
0,1(T)\end{array}$ & $\begin{array}{c}\text { Rp Magnetita } \\
(\%)\end{array}$ & $\begin{array}{c}\text { Rp Estimación } \\
(\%)\end{array}$ & $\begin{array}{c}\text { Satmagan } \\
(\%)\end{array}$ \\
\hline Desviación absoluta media (\%) & 3,33 & 3,77 & 3,13 & 3,99 & 3,87 \\
\hline Error medio cuadrado (\%) & 14,16 & 31,85 & 15,35 & 25,52 & 22,68 \\
\hline Porcentaje de error medio absoluto (\%) & 7,77 & 9,60 & 7,01 & 8,87 & 8,62 \\
\hline
\end{tabular}

Junto con el análisis descriptivo, se realizó un análisis ANOVA de comparación de medias para las diferentes recuperaciones obtenidas por los diferentes métodos, aplicando el software estadístico Statgraphics Centurion XV. Es por ello que en la Tabla 5 se presenta la identificación utilizada para realizar el análisis, mientras que en la Tabla 6 se presentan los resultados de este.

Tabla 5: Identificación utilizada para análisis en Statgraphics

\begin{tabular}{|l|c|}
\hline \multicolumn{1}{|c|}{ Método } & Identificación \\
\hline Rp por nivel de estanque & A \\
\hline Rp por ajuste lineal & B \\
\hline Rp DTT 0,1 (T) & C \\
\hline Rp DTT 0,4 (T) & D \\
\hline SATMAGAN & E \\
\hline Rp magnetita & F \\
\hline
\end{tabular}


Tabla 6: Resultados de análisis ANOVA para los diferentes métodos evaluados

\begin{tabular}{|l|c|c|c|c|}
\hline \multicolumn{1}{|c|}{ Fuente } & Suma de Cuadrados & Grado de libertad & Cuadrado Medio & Valor-P \\
\hline Entre grupos & 420,44 & 5,00 & 84,09 & 0,00 \\
\hline Intra grupos & 1258,80 & 156,00 & 8,07 & - \\
\hline Total (Corregido) & 1679,24 & 161,00 & - & - \\
\hline
\end{tabular}

Es posible observar que el valor-P presenta un valor de 0,0 , por lo cual se rechaza la hipótesis de igualdad de medias, debido a que los diferentes métodos evaluados presentan medias estadísticamente diferentes, razón por la cual se realizó un análisis de rangos múltiples, utilizando el método de Tukey, para verificar cuáles combinaciones de resultados presentan diferencias significativas. En la Tabla 7 se presentan las combinaciones de los diferentes métodos evaluados con respecto al valor real de recuperación en peso obtenido en planta, donde es posible inferir que las recuperaciones en peso calculadas a través del ensayo DTT realizado a 0,4 Tesla, junto con la recuperación calculada en base a magnetita presentan similitudes estadísticas en sus medias, con respecto al valor real con una confianza del $95 \%$. De esta manera se valida que efectivamente es posible obtener un parámetro de recuperación en peso, relacionando la información que entregan los diferentes ensayos

Tabla 7: Prueba de rangos múltiples para recuperación por tratamiento

\begin{tabular}{|c|c|c|c|}
\hline Contraste & Significativa & Diferencia & +/- Limites \\
\hline A - B & $*$ & 2,9733 & 2,2308 \\
\hline A - C & $*$ & 3,7696 & 2,2308 \\
\hline A - D & & $-0,7300$ & 2,2308 \\
\hline A - E & $*$ & 2,5996 & 2,2308 \\
\hline A - F & & 2,0026 & 2,2308 \\
\hline * Indica diferencia significativa \\
\hline
\end{tabular}

\section{CONCLUSIONES}

Los resultados obtenidos en este trabajo se pueden establecer las siguientes conclusiones: 1) Los ensayos DTT realizados a muestras provenientes de Planta CNN, Planta Magnetita y Planta Los Colorados, solo se observó que para las muestras de medio y bajo magnetismo provenientes de Planta CNN, bajo los 0,2 Tesla, se presentan diferencias considerables en la recuperación en peso (Rp DTT); 2) La cantidad de magnetita calculada a través de la balanza de saturación magnética Satmagan, guarda estrecha relación con respecto al cálculo realizado a través del análisis químico óxido ferroso. Se observó que con el método químico se obtienen cantidades levemente mayores de magnetita en las muestras, dado que dicho método considera todo el ion ferroso obtenido en el análisis químico como magnetita; 3) Para las muestras de Planta CNN, se determinó que solo las muestras de alto magnetismo presentan una relación entre la recuperación en peso DTT (Rp DTT) y el \% de magnetita obtenido por Satmagan, mientras que para las muestras de mediano y bajo magnetismo no existe relación para estos análisis; 4) Se pudo comprobar que, efectivamente, la recuperación en peso calculada en base a magnetita sirve como estimador para la recuperación en peso global del circuito de Planta CNN, considerando los errores calculados, junto con el análisis ANOVA y el análisis de rangos múltiples, en los cuales se pudo determinar que no existen diferencias estadísticamente significativas entre el valor de recuperación en peso obtenido por planta con respecto al calculado en base a magnetita.

\section{NOTACIÓN}

$\mathrm{Rp}_{\text {Magnetita }}$ : Recuperación en peso calculada respecto a la magnetita en el concentrado DTT, \%

$\mathrm{FeO}_{\mathrm{CDTT}}$ : \% de $\mathrm{FeO}$ presente en el concentrado DTT, \%

$\mathrm{Rp}_{\mathrm{DTT}}$ : Recuperación en peso obtenida en ensayo Davis Tube Tester (DTT), \%

$\mathrm{C}_{1}$ : Relación estequiométrica ( $\mathrm{PM} \mathrm{Fe}_{3} \mathrm{O}_{4} / \mathrm{PM} \mathrm{FeO}$ )

$\mathrm{C}_{2}$ : Factor de impurezas presente en el concentrado DTT, \% 


\section{REFERENCIAS}

Abdel Haffez, G., y Abu-Ali, M., Evaluation and Optimization of Operating Parameters Affecting the Davis Tube Magnetic Tester by Using $2^{\text {nd }}$ Factorial Design, https://doi.org/10.3139/120.110350, Materials Testing, 54(6), 437-443 (2012)

Ahmed, M. M., Statistical Design Application and Analysis of Separation Efficiency in Davis Tube Tester, DOI: 10.21608/JESAUN.2010.125562, Journal of Engineering Sciences, 38(4), 1047-1058, (2010)

Baawuah, E., Kelsey, C., Addai-Mensah, J., y Skinner, W., Assessing the performance of a novel pneumatic magnetic separator for the beneficiation of magnetite ore, DOI:10.3390/min10090759, Miner. Eng., 156, 106483 (2020)

Du Breuil, C., Perroton, A., y otros cinco autores., Iron Oxides Evolution along the Lateritic Profile of Mabounié Carbonatite (Gabon): A Key Point to Understand Magnetic Separation Processes, Mineral Resources in a Sustainable World, 13th SGA Biennial Meeting, Proceedings, 4, 1369 -1372 (2015)

Dwari, R.K., Rao, D.S., y Reddy, P.S.R., Magnetic separation studies for a low-grade siliceous iron ore sample, DOI: $\{10.1016 / j . j$ ijmst.2013.01.001\}, Int. J. Miner. Sci. Technol., 23(1), 1-5 (2013)

Dworzanowskl, D., Maximizing the Recovery of Fine Iron Ore Using Magnetic Separation, J. S. Afr. Inst. Min. Metall., ISSN 2225-6253, 112(3), 197-202 (2012)

Ersayin, S., Low intensity magnetic separator modelling: A pseudo liberation approach, https://doi.org/10.1179/037195504225006579, Mineral Processing and Extractive Metallurgy, 113(3), 167-174 (2004)

Ge, W., Encinas, A., Araujo, E., y Song, S., Magnetic matrices used in high gradient magnetic separation (HGMS): A review, https://doi.org/10.1016/j.rinp.2017.10.055, Results in Physics, 7, 4278-4286 (2017)

$\mathrm{Hu}, \mathrm{H}$., Tang, Y., y otros cuatro autores, The effect of copper on iron reduction and its application to the determination of total iron content in iron and copper ores by potassium dichromate titration, DOI: 10.1016/j.talanta.2014.03.008, Talanta, $125,425-431$ (2014)

Issa, B., Obaidat, I. M., Albiss, B. A., y Haik, Y., Magnetic nanoparticles: surface effects and properties related to biomedicine applications, https://doi.org/10.3390/ijms141121266, International Journal of Molecular Sciences, 14(11), 21266-21305 (2013)

Khokhulya, M., Fomin, A., y Alekseeva, S., Recovery of magnetite-hematite concentrate from iron ore tailings, https://doi.org/10.1051/e3sconf/202124701042, E3S Web of Conferences, 247, 01042 (2021)

Lund, C., Lamberg, P., y Lindberg, T., Practical way to quantify minerals from chemical assays at Malmberget iron ore operations - An important tool for the geometallurgical program, https://doi.org/10.1016/j.mineng.2013.04.005, Miner. Eng., 49, 7-16 (2013)

Stener, J. F., Carlson, J. E., Pålsson, B., y Sand, A., Direct Measurement of Internal Material Flow in a Bench Scale wet low-intensity Magnetic Separator, https://doi.org/10.1016/j.mineng.2015.10.021, Miner. Eng., 91, 55-65 (2016)

Valderrama, L., Tapia, J., Gómez, O., y Santander, M., Recuperación de apatita desde relaves en columna de flotación, http://dx.doi.org/10.4067/S0718-07642020000100321, Información Tecnológica, 31(1), 321-328 (2020)

Xiong, D., Lu, L., y Holmes, R. J., Iron Ore Mineralogy, Processing and Environmental Sustainability. Lu, L., Ed., Woodhead Publishing, ISBN 9781782421566, Cambridge, UK (2015) 